\title{
LA ESTRATEGIA ADM DE LA UE Y LAS ARMAS QUÍMICAS: UNA COLABORACIÓN EN LA ENCRUCIJADA
}

\author{
Fernando Borredá ${ }^{1}$ \\ Ministerio de Asuntos Exteriores de España (MAEC)
}

\begin{abstract}
Resumen:
El 12 de diciembre del 2003, la Unión Europea adoptó la Estrategia Contra la Proliferación de Armas de Destrucción Masiva (Estrategia ADM) que se ha materializado bajo el principio de "multilateralismo efectivo". Respecto a la proliferación de las armas químicas, las iniciativas de la UE se han llevado a cabo, principalmente, dentro del régimen establecido por la Convención para la Prohibición del Desarrollo, la Producción, el Almacenamiento y el Uso, así como de su Destrucción (CAQ) mediante acuerdos de contribución con la Organización para la Prohibición de las Armas Químicas (OPAQ). No obstante, el contexto de seguridad ha evolucionado considerablemente desde que entró en vigor el régimen de la CAQ en 1997. Además el régimen establecido por la CAQ presenta vulnerabilidades, bien estructurales o bien de implantación, que pueden cuestionar su efectividad. El propósito de este artículo es analizar la evolución (o falta de ella) de la política de la UE respecto la proliferación de armas químicas en coherencia con la Estrategia ADM a la vista de tanto los cambios de contexto de seguridad, como las debilidades del régimen de la CAQ en el que se apoya. Este análisis nos llevara a concluir que, en la medida que la UE ha confiado su política de las armas químicas a la OPAQ, se hace dependiente de las vulnerabilidades y de la evolución del régimen de CAQ, lo que puede llegar a ir en detrimento del su propia estrategia inicial.
\end{abstract}

Palabras clave: Unión Europea, Armas de Destrucción Masiva, Estrategia ADM, armas químicas, Organización para la Prohibición de las Armas Químicas.

Title in English: "The EU's MDW Strategy and the Chemical Weapons: Cooperation at the Crossroads".

Abstract:

On 12 December 2003, the European Union adopted the Strategy against proliferation of Weapons of Mass Destruction (WMD Strategy) that has been materialized under the principle of "effective multilateralism". Regarding the proliferation of chemical weapons, the EU initiatives have been carried out mainly within the regime established by the Convention on the Prohibition of the Development, Production, Stockpiling and Use, and on their Destruction (CWC) through contribution agreements with the Organization for the Prohibition of Chemical Weapons $(O P C W)$. However, the security context has evolved considerably since the entry into force of the CWC regime in 1997. Moreover, the regime established by the CWC has vulnerabilities, due to structural or implementation reasons, which may question their effectiveness. The purpose of this article is to analyze the evolution (or lack thereof) of the EU policy on chemical weapons proliferation consistent with the WMD Strategy in view of both, the security context evolution and the vulnerabilities of the CWC regime. This analysis leads us to conclude that, to the extent that the EU has entrusted its policy of chemical weapons to the OPCW, becomes dependent on the vulnerabilities and the evolution of the CWC regime, which can become detrimental to the its own main strategy on WMD.

Keywords: European Union, Massive Destruction Weapons, ADM Strategy, chemical arms, Organization for the Prohibition of Chemical Weapons.

Copyright $\odot$ UNISCI, 2012.

The views expressed in these articles are those of the authors, and do not necessarily reflect the views of UNISCI. Las opiniones expresadas en estos artículos son propias de sus autores, y no reflejan necesariamente la opinión de UNISCI.

\footnotetext{
${ }^{1}$ Fernando Borredá Juste is Head of Disarmament and Non proliferation Area at the Spanish Ministry of Foreign Affairs. He holds a Masters degree in International Security and is currently completing a $\mathrm{PhD}$ dissertation on the international regime on chemical weapons at the UNED (Spanish Distance University). He has previously worked as a chemical weapons inspector for the Organisation for the Prohibition of Chemical Weapons in the Hague and as conventional weapons inspector for the Spanish Armed Forces.

E-Mail: fborreda@yahoo.es.
}

http://dx.doi.org/10.5209/rev_UNIS.2012.n30.4070 


\section{Introducción}

El 12 de diciembre del 2003, la Unión Europea presentó la primera (y única) Estrategia Europea de Seguridad "Una Europa Segura en un Mundo Mejor" (EES) ${ }^{2}$, así como los principios básicos de la UE contra la proliferación reflejados en la Estrategia Contra la Proliferación de Armas de Destrucción Masiva (Estrategia ADM) ${ }^{3}$. La EES de la Unión Europea destaca la proliferación de las ADM como la amenaza potencial más grave para la seguridad, por añadidura, su combinación junto la descomposición de los Estados poseedores de ADM constituye una amenaza más radical si cabe. La combinación de estas dos graves amenazas se ha hecho resurgir el problema de las armas químicas como se ha puesto de manifiesto en las revoluciones de la "primavera árabe" en Estados poseedores de armas químicas ${ }^{4}$. En consecuencia, la evolución del contexto de seguridad, junto con las carencias en la implantación y los nuevos retos al régimen de la CAQ, hacen necesario cuestionarse sobre la política de las armas químicas de la UE en relación con la Estrategia ADM.

Este documento analiza la evolución de la política de las armas químicas de la UE, tras casi diez años de aquella decisión estratégica, teniendo en cuenta las debilidades del régimen de la CAQ sobre el que apoya su "multilateralismo efectivo", así como las consecuencias y efectividad de su relación con la OPAQ respecto a los principios establecidos por su Estrategia de ADM. Así, en la segunda sección, se detallan y analizan los instrumentos legales, en forma de decisiones del Consejo y acuerdos institucionales como instrumentos para materializar la Estrategia ADM, particularmente con el régimen de la CAQ. La III sección presenta las carencias y desafíos del régimen de las armas químicas. En la sección IV se evalúa la efectividad de la acción de la UE a la vista de las amenazas y vulnerabilidades del régimen de la CAQ. Finalmente, la sección V extrae conclusiones en cuanto a la evolución de la política de las armas químicas de la UE, así como las consecuencias que las vulnerabilidades del régimen de la CAQ tienen sobre la Estrategia de la UE, terminado con una recomendación al respecto.

\section{Los instrumentos de la UE en el marco de la estrategia ADM en el ámbito de las armas químicas}

Los atentados del 11 de Septiembre de 2011 trajeron, entre otras, la inquietud del "todo es posible" por el riesgo de empleo de cualquier tipo de armas por parte de terroristas para conseguir sus objetivos. Esta posibilidad encontraba su paradigma en el la utilización de Armas de Destrucción Masiva por su capacidad para sembrar el terror y multiplicar los daños, haciendo resurgir el problema de la proliferación de las armas nucleares, biológicas y, como el caso que nos ocupa, químicas.

El 12 de Diciembre del 2003, la Unión Europea adoptó los dos principales documentos contra la proliferación de ADM, tanto la Estrategia Europea de Seguridad (EES), como la Estrategia Contra la Proliferación de Armas de Destrucción Masiva (Estrategia ADM).

\footnotetext{
${ }^{2}$ Council of the European Union: "A Secure Europe in a Better World: European Security Strategy", Bruselas (12 de Diciembre 2003).

3 Council of the European Union: "EU Strategy against Proliferation of Weapons of Mass Destruction", 15708/03, Bruselas (10 de Diciembre 2003).

${ }^{4}$ Blitz, James: "Fears grow over Syria's chemical arms", Financial Times, 15 de julio 2012; "Libya's Muammar Gaddafi had chemical weapon cache", BBC News, 20 de enero 2012, en http://www.bbc.co.uk/news/worldafrica-16650192.
} 
Para conseguir sus objetivos, la UE decide adoptar un enfoque basado en el "multilateralismo efectivo" sobre tres elementos: Multilateralismo, mediante el fomento del sistema de tratados internacionales. La prevención, mediante la promoción de un entorno regional e internacional estable combatiendo las causas subyacentes a la proliferación (prestando especial atención a la cuenca mediterránea) y, finalmente, la cooperación internacional, mediante el estrechamiento de la cooperación con los socios clave (Estados Unidos, Rusia, Japón y Canadá) ${ }^{5}$.

A este respecto, la Estrategia ADM introdujo un Plan de Acción revisable (Living Action Plan $)^{6}$ desarrollado en cuatro líneas de acción. La primera, de carácter pro-activo, tiene como objeto actuar con decisión contra los proliferadores. En esta línea se establecen varias medidas: primero, elevar los principales tratados, acuerdos y acuerdos de verificación a la categoría de universales y, si fuere necesario, reforzarlos. Segundo, favorecer el papel del Consejo de Seguridad de las Naciones Unidas. Tercero, incrementar el apoyo político, financiero y técnico de los sistemas de verificación. Cuarto, reforzar las políticas y la práctica en materia de control de las exportaciones. Quinto, incrementar las medidas de seguridad que impiden el acceso no autorizado a materiales, equipos y conocimientos sensibles en materia de proliferación en la Unión Europea. Finalmente, sexto, reforzar la localización, el control y la interceptación de tráficos ilícitos.

La segunda línea de acción, se puede considerar como la parte de incentivos dentro de la comúnmente llamada política del "palo y la zanahoria". El objetivo es fomentar un entorno internacional y regional estable mediante, primero, el refuerzo a los programas comunitarios de cooperación y ayuda al desarme, así como al control y a las medidas de seguridad de materiales, instalaciones y conocimientos sensibles (particularmente los programas de las Federación Rusa); segundo, integrar la no proliferación de ADM a las actividades y políticas de la UE mediante la inclusión de la "cláusula de no proliferación" en otros acuerdos con terceros países, con el fin de incrementar su eficacia y representado la característica de "poder civil" de la UE, capaz de utilizar su potencia económica y financiera como instrumento de poder $^{7}$.

Una tercera línea de acción, con el propósito de cooperar estrechamente con los Estados Unidos y otros socios clave. Finalmente, la cuarta línea crear las estructuras necesarias en la Unión, principalmente la creación de una unidad específica y centro de seguimiento de la aplicación de la Estrategia.

En el ámbito de las armas químicas, la Estrategia de ADM inicialmente se materializó con la primera Acción Conjunta con la OPAQ en el año 2004 ${ }^{8}$. Esta Acción Conjunta implicaba un apoyo financiero total 1,8 millones de euros para el año 2005, sujeto a tres proyectos/propósitos: promoción de la Universalidad de la CAQ, en particular facilitar el acceso de Estados no Parte (0,2 Mill. de euros), apoyo al establecimiento e implantación de CAQ en los Estados Parte (0,4 Mill. de euros) y cooperación internacional en el ámbito de los usos pacíficos de la química (1 Mill. de euros).

\footnotetext{
${ }^{5}$ Council of the European Union: "WMD Strategy”, Chapter II.

${ }^{6}$ Ibid., Chapter III.

7 Ham, Peter Van: “The European Union's WMD Strategy and the CFSP: a critical analysis”, EU NonProliferation Consortium Non-Proliferation Papers, no. 2 (Sept. 2011), p. 4.

${ }^{8}$ Council of the European Union: "On support for OPCW activities in the framework of the implementation of the EU Strategy against Proliferation of Weapons of Mass Destruction”, Council Joint Action 2004/797/CFSP (22 de Noviembre 2004).
} 
Respecto a la promoción de universalidad, el proyecto implicaba la organización de seminarios en el año 2005 para Estados no Parte en el Caribe ${ }^{9}$, en África ${ }^{10}$, Oriente Próximo ${ }^{11}$ y Asia ${ }^{12}$. En el año siguiente se repitió el mismo esquema de Acción Conjunta ${ }^{13}$ con una financiación 1,6 millones de euros.

No obstante, los atentados de Madrid y Londres pusieron de relieve los nuevos desafíos que plantean los agentes no estatales, la necesidad imperiosa de impedir que los terroristas adquieran armas de destrucción masiva o materiales relacionados con ellas y, por tanto, la importancia extrema de intensificar los esfuerzos por aplicar la Estrategia de la UE contra la proliferación de armas de destrucción masiva. Como consecuencia, en diciembre de 2006, el Consejo aprobó un "documento de reflexión"14 para mejorar la eficacia y la coherencia de la Estrategia de la UE contra la proliferación de ADM. El propósito del documento era establecer un método de trabajo cooperativo que permitiese al Secretario del Consejo y Alto Representante, a los Servicios de la Comisión y a los Estados miembros trabajar conjuntamente y obtener mejores sinergias a la vez que evitar duplicaciones. Ello supuso la creación de un centro de seguimiento, una unidad específica que sirva de centro de control y que se encargue del seguimiento de la aplicación coherente de la Estrategia de la UE, así como de la recogida de información y datos, en colaboración con el Centro de Situación. Este centro de seguimiento se crearía en la Secretaría del Consejo y la Comisión participaría plenamente en sus trabajos.

El “documento de reflexión” se materializó en la Acción Conjunta del año $2007^{15}(1,7$ millones de euros). Aunque no supuso un incremento de presupuesto, si que reflejaba un importante cambio tanto en los propósitos, como en las prioridades de los proyectos. En este sentido, se aumentó el numero de proyectos, pasando de tres a siete, añadiendo proyectos hacia nuevas áreas como la implantación nacional de la CAQ, particularmente respecto las declaraciones y legislación, la cooperación con la industria y, principalmente, la asistencia y protección contra las armas químicas (Art. X).

En el año 2008, durante la presidencia francesa de la UE, con las primeras voces cuestionando la implantación real de Estrategia del $\mathrm{UE}^{16} \mathrm{y}$, tras las conclusiones del seminario específico respecto el problema de la no proliferación en París del 15-16 de Julio, la política de la UE respecto a las ADM adquirió un nuevo impulso a través de las Nuevas Líneas de Acción de la UE en combatir las ADM y sus sistemas de lanzamiento ${ }^{17}$. La amenaza de las

\footnotetext{
${ }^{9}$ Incluyendo invitaciones Antigua y Barbudas, Bahamas, Barbados, Republica Dominicana, Haití, Honduras y Grenada.

${ }^{10}$ Incluyendo invitaciones Angola, República Centro Africana, las islas Comoros, Congo, República Democrática del Congo, Djibouti, Egipto, Guinea-Bissau, Liberia, Madagascar, Sierra Leona, y Somalia.

${ }^{11}$ Incluyendo invitaciones a Egipto, Irak, Israel, Líbano y Siria.

${ }^{12}$ Incluyendo invitaciones a Bután, Camboya, República Popular Democrática de Corea, Irak, Líbano, Myanmar, Niue, Siria, Islas Salomón y Vanuatu.

${ }^{13}$ Council of the European Union: Council Joint Action 2005/913/CFSP, "On support for OPCW activities in the framework of the implementation of the EU Strategy against Proliferation of Weapons of Mass Destruction", 12 diciembre de 2005.

${ }^{14}$ Council of European Union, "EU Strategy against the proliferation of WMD: Monitoring and enhancing consistent implementation" (12 diciembre de 2006).

${ }^{15}$ Council of the European Union: "On support for OPCW activities in the framework of the implementation of the EU Strategy against Proliferation of Weapons of Mass Destruction”, Council Joint Action 2007/185/CFSP (19 marzo de 2007).

${ }^{16}$ Oliver, Meier: “The EU's Nonproliferation Efforts: Limited Success”, Arms Control Today (May 2008), at http://www.armscontrol.org/act/2008 05/OliverFeature\#28.

${ }^{17}$ Council of European Union: "New Lines for Action by the European Union in Combating the Proliferation of Weapons of Mass Destruction and their Delivery Systems”, Bruselas, 17 diciembre 2008.
} 
$\mathrm{ADM}$ en manos de terroristas/actores no estatales quedó definida como "el desafío más grande a la seguridad que los europeos pueden llegar a enfrentarse", adquiriendo "la más alta prioridad" la lucha contra la proliferación de ADM. En consecuencia, las Nuevas Líneas de Acción tuvieron un propósito más operacional que el "multilateralismo efectivo" establecido en la Estrategia de ADM del 2003.

Así se establecieron cuatro objetivos con el propósito de ser implementados antes del final del año 2010, en primer lugar, elevar las medidas de no proliferación pasando de ser un asunto de seguridad fundamental a tener un carácter de prioridad transversal (cross cutting prorities) afectando a más ámbitos que los estrictamente relacionados con la seguridad (sociedad civil, industria, académicos...). En segundo lugar, identificar las mejores prácticas e implementarlas, tercero, mejorar la coordinación y movilización de las políticas de los Estados y la UE, y finalmente, identificar las áreas de mejora de la política de la UE. Para ello se establecieron cinco líneas: Conocimiento y Anticipación, Prevención, Impedimento y Detención, Cooperación y Apoyo, finalmente, Coordinación.

En cuanto a las armas químicas se refiere, las Nuevas líneas de Acción se han materializado en tres direcciones principalmente. En primer lugar, a la mejora de la capacidades de protección y asistencia contra la amenaza QBRN (químicas, Bacteriológicas, Radiológicas y Nucleares), más conocidas como "bombas sucias". En este sentido, destaca la creación del Plan de Acción QBRN con objeto de incrementar la capacidad de prevención, detección, preparación y respuesta particularmente en regiones menos desarrolladas, que ha conllevado la creación de Centros de Excelencia QBRN en África, en la región del Mar Negro, en el Centro y Sur-Este de Asia y en el Oriente Próximo, contra la amenaza QBRN en regiones menos desarrolladas (25-30 millones de euros). En segundo lugar, la lucha contra el tráfico ilícito QBRN estableciendo un régimen de control a la exportación y tecnología de doble uso bajo el cual equipos y materiales de doble uso (listados en la CAQ) no pueden salir del territorio europeo sin una autorización específica ${ }^{18}$. Finalmente, estrechando la cooperación con la OPAQ mediante los Acuerdos de Contribución de los años $2009^{19}$ y $2012^{20}$ que garantizan unos fondos de 2,1 y 2,14 Mill. de euros respectivamente.

La diferencia principal de las dos últimas contribuciones respecto las respectivas del periodo 2004-2008 se encuentra en la forma de asignación de los recursos. Al igual que muchas contribuciones nacionales, la contribución de la UE durante los años 2004-2008 fue específica para cada proyecto y sus beneficiarios. Por ejemplo, la transferencia de equipos a los laboratorios de África, el desarrollo de la base de datos de sustancias químicas sujetas a control, o la ayudas a la construcción de capacidades nacionales de protección contra las armas químicas. Si bien esta asignación inflexible puede ocasionar una disminución de la capacidad organizativa de la Secretaría Técnica de la OPAQ, y causar desequilibrios en áreas temáticas o países ${ }^{21}$, facilita la valoración por parte de la sociedad civil de los criterios de

\footnotetext{
${ }^{18}$ Council of the European Union: "Setting up a Community regime for the control of exports, transfer, brokering and transit of dual-use items", Council Regulation (EC) No 428/2009, 5 mayo 2009.

${ }^{19}$ Council of the European Union: "On support for OPCW activities in the framework of the implementation of the EU Strategy against Proliferation of Weapons of Mass Destruction”, Council Decision, 2009/569/CFSP, 27 de julio 2009.

${ }^{20}$ Council of the European Union: "Council Decision 2012/166/CFSP, In support of activities of the Organisation for the Prohibition of Chemical Weapons (OPCW) in the framework of the implementation of the EU Strategy against Proliferation of Weapons of Mass Destruction”, 23 de marzo 2012.

${ }^{21}$ Grip, Lina: "Assessing Selected European Union External Assistance and Cooperation Projects on WMD Non-Proliferation”, EU Non-Proliferacion Consortium, Non-Proliferation Papers, no. 6 (Dic. 2011), p. 7.
} 
asignación de los recursos y la coherencia con los objetivos estratégicos, al poder cotejar las cantidades asignadas con las prioridades propuestas.

Por otra parte, la UE cuenta con otros instrumentos a su disposición para alcanzar el objetivo de mejorar participación en los instrumentos multilaterales de no proliferación, la señalada "cláusula de no proliferación". Iniciada en 2003, la cláusula tiene el propósito de integrar las políticas de no proliferación dentro del amplio paraguas de las relaciones de la UE. Básicamente, la cláusula de no proliferación es una cláusula a ser incluida como condicionante en los acuerdos (nuevos o revisados) de la UE con terceros países. Respecto su impacto en el ámbito de las armas químicas, la inclusión en los acuerdos de la UE con los respectivos terceros países ha sido aceptada generalmente, influyendo a la hora de seleccionar países beneficiarios de ayuda europea que se materializó en seminarios, visitas de asistencia y apoyo de equipos. Concretamente, de los 27 países que accedieron a la CAQ desde diciembre del 2003, 14 fueron beneficiarios de la ayuda de la UE condicionada a la inclusión de la cláusula de no proliferación ${ }^{22}$.

Adicionalmente, la UE ha desarrollado una intensa actividad en cuanto a su apoyo a la destrucción del arsenal de las armas químicas en la Federación Rusa. En primer lugar se estableció de manera bilateral en $1999^{23}$ con 8,9 millones de Eur. para financiar la instalación de destrucción de armas químicas de Gorny. Posteriormente, en 2001 con 6 Mill. de euros apoyo a Agencia Rusa de Municiones y financiar la infraestructura de la instalación de destrucción de Shchu'ye ${ }^{24}$. A partir del año 2002, dentro del Grupo del G8 (G8 Global Partnership) la Comisión Europea asignó 6 Mill. de euros para reconvertir instalaciones de producción de armas químicas a fines pacíficos, en el año 2003 se asignaron 0,7 Mill. de euros en apoyo de equipo para la instalación de destrucción de Kambarca ${ }^{25}$. Finalmente, en el 2007, se financió con 3.14 Mill. de euros a la construcción de la instalación de Shchu'ye ${ }^{26}$. En total, durante este periodo, se comprometieron 14,79 Mill. de Eur., de los cuales solamente se consumieron 10 Mill. de euros por diversos problemas de aplicación a nivel operacional. Aunque la ayuda europea no ha impedido que la Federación Rusa no pueda completar su destrucción antes de la fecha final programada por la CAQ (abril 2012) ${ }^{27}$, no cabe ninguna duda que la ayuda europea fue fundamental para iniciar el proyecto de destrucción en la Federación Rusa hasta que su delicada situación económica se solucionase en el periodo 2005-2007 ${ }^{28}$ y pudiera asumir los costes de su propia destrucción.

\footnotetext{
${ }^{22}$ Council of the European Union: "Note on the implementation of the WMD clause", 19 de Enero 2009.

23 Council of the European Union: "Establishing a European Union Cooperation Programme for Nonproliferation and Disarmament in the Russian Federation", Council Joint Action, 1999/878/CFSP, 17 de Diciembre 1999.

${ }^{24}$ Council of the European Union: "Implementing Joint Action 1999/878/CFSP with a view to contributing to the European Union Cooperation Programme for Non-proliferation and Disarmament in the Russian Federation", Council Decision 2001/493/CFSP, 25 de Junio 2001.

${ }^{25}$ Council of the European Union: "Implementing Joint Action 2003/472/CFSP with a view to contributing to the European Union cooperation programme for non-proliferation and disarmament in the Russian Federation", Council Decision, 2003/874/CFSP, 8 de Diciembre 2003.

${ }^{26}$ Council of the European Union: "In support of chemical weapons destruction in the Russian Federation in the framework of the EU Strategy against Proliferation of Weapons of Mass Destruction", Council Joint Action, 2007/178/CFSP, 19 de Marzo 2007.

${ }^{27}$ Aproximadamente un 50\% de arsenal. OPCW Conference of State Parties: "Report of the OPCW on the Implementation of the Convention on the Prohibition of the Development, Production, Stockpiling and Use of Chemical Weapons and on their Destruction in 2010", C-16/4, 30 Noviembre 2011.

${ }^{28}$ Hart, John: "Assistance for the destruction of chemical weapons in the Russian Federation: political and technical aspects", Background paper 5, Conference on Strengthening European Action on WMD Nonproliferation and Disarmament: How Can Community Instruments Contribute?, Bruselas, 7-8 diciembre 2005.
} 


\section{Los desafíos actuales al régimen de las armas químicas}

Aunque la validez de un régimen de seguridad pueda ser medida empíricamente en términos de resultados exactos, como el caso de la destrucción completa de un tipo de armas, no significa que los Estados Parte hayan internalizado todos los principios y normas del régimen, de forma que la efectividad pueda quedar en entredicho. En consecuencia, a pesar de que se hayan destruido una mayor parte de los arsenales de armas químicas, resulta necesario identificar las vulnerabilidades del régimen para evaluar su efectividad real. Así, los principales puntos débiles del régimen de la CAQ se pueden dividir en cinco tipos generales: las declaraciones y su implantación nacional, el proceso de destrucción y desarme, las inspecciones industriales junto los avances en la ciencia y tecnología, finalmente, la universalidad.

A este respecto, hemos podido observar un resultado desigual en cuanto al nivel de cumplimiento de las disposiciones de la CAQ en las cuatro áreas normativas principales, esto es, la norma de información o declaraciones como consecuencia de una implantación no eficiente de las Autoridades Nacionales, la norma de destrucción con los retrasos en la consecución de las destrucciones y la norma de inspección, particularmente las inspecciones industriales a la vista de los avances de la ciencia y tecnología y la universalidad con el problema de los estados fallidos.

Todo el proceso de verificación tiene su base en las declaraciones, de forma que el papel del órgano de la OPAQ que confecciona las mismas, la Autoridad Nacional adquiere un importante peso específico. Por este motivo, algunos analistas consideran, incluso desde antes de la propia llegada al acuerdo, un error descansar la responsabilidad de la verificación únicamente en la Secretaria Técnica ${ }^{29}$. Así, el papel de las Autoridades Nacionales adquiere mayor relevancia si se tiene en cuenta, en un campo tan dinámico como la química y la biología, la aparición de nuevos compuestos con características tóxicas sujetos a declaración o innovadores procedimientos de producción (microreactores, nanotecnología, química combinatoria...), que pudieran pasar desapercibidos por la Secretaría Técnica. Bajo esta perspectiva, la Autoridad Nacional constituye el escalón básico, y fundamental, del proceso de verificación a nivel nacional, pero cuya efectividad tiene consecuencias sobre el régimen internacional de verificación por la posibilidad de restringir sus actividades solamente a las sustancias químicas establecidas en las listas, o aplicar una interpretación más amplia de acorde con el Criterio General de Propósito ${ }^{30}$. A este respecto, resulta también imprescindible el apoyo de la industria, especialmente en los países en vías de desarrollo, donde la mayor parte de la producción se ha trasladado por motivo de costes y oportunidad de mercados emergentes. Aumentar la cooperación industria-ciencia-instituciones se hace más necesario a la vista de los cambios geográficos y de los nuevos avances de la ciencia ${ }^{31}$.

En cuanto a la norma de información, hemos podido observar que la cuestión de las declaraciones industriales ha presentado una significativa mejora en el grado de implantación del régimen, tras unos inicios considerados como pobres y en el margen de lo aceptable ${ }^{32}$.

\footnotetext{
${ }^{29}$ Robinson, Julian Perry: "The verification system for the Chemical Weapons Convention", The Hague Academy of International Law, the Convention on the Prohibition and Elimination of Chemical Weapons: A Breakthrough in Multilateral Disarmament, Martinus Nijhoff, Dordrecht (1995), p. 490.

${ }^{30}$ Feakes, Daniel: "Evaluating the CWC Verification System", Disarmament Forum, no. 4 (2002), p. 15.

${ }^{31}$ Crowley, Michael: "Chemical weapons disarmament and the future of OPCW", Conference report (18- 20 March 2011), p.12.

${ }^{32}$ Kelle, Alexander: "Assessing the Effectiveness of Security Regimes - The Chemical Weapons Control Regime's First Six Years of Operation”, International Politics, nº. 41(2004), p. 230. Por añadidura, la cuestión
} 
Además, la mejora ha venido acompañada de cambio en la aproximación a los asuntos, de un enfoque cuantitativo a uno cualitativo, lo que demuestra el alcance del proceso, donde la fase de volumen general ha dejado paso a la definición y perfeccionamiento del mismo ${ }^{33}$. No obstante, la norma esta menos desarrollada en las áreas de control de la exportación y a las actividades encaminadas a asistir, incentivar, o comprometerse en actividades prohibidas a nivel nacional. En este ámbito se hace necesaria una evolución, encontrando nuevas prácticas a nivel Autoridad Nacional o Secretaría Técnica. El hecho de que aquellas áreas estén apoyadas tan sólo marginalmente por las actividades de verificación implica la necesidad de apoyos externos para mejorar las capacidades de la OPAQ en esta área, bien mediante acuerdos que impulsen una mejora de las capacidades de las Autoridades Nacionales, mediante la demanda de más información respecto cómo éstas llevan a cabo sus obligaciones de verificación a nivel nacional y estableciendo nuevas actividades de control, siempre dentro de la legalidad de la Convención, o bien estableciendo normas adicionales de la UE que complementen las carencias de la OPAQ.

En relación con la norma de desarme, el proceso de destrucción se ha revelado como más largo y extraordinariamente más costoso de lo que se había estimado durante la redacción de la Convención. Esta dificultad ha provocado varias situaciones con consecuencias favorables y desfavorables al régimen. Por el lado positivo, la garantía en la extensión de las fechas intermedias y finales a todos los Estados Parte poseedores por igual, a pesar de la enorme diferencia de volumen de arsenal a destruir de las dos superpotencias (Estados Unidos y la Federación Rusa), respecto del resto de los Estados Poseedores (India, "un Estado Parte", Libia y Albania), refuerza la característica "no discriminatoria" de la CAQ. Además, ha estimulado la cooperación internacional para facilitar la destrucción mediante ayudas económicas y tecnológicas a Estados Parte poseedores en dificultades financieras ya señaladas como a la Federación Rusa o Albania por parte de la UE. En cuanto al incumplimiento de la norma de destrucción por el retraso en las fechas finales de destrucción completa, puede que no resulte tan significativo si no implica un riesgo sobre los principios de régimen, especialmente cuando se ha demostrado la intención inequívoca de los Estados Poseedores de destruir su arsenal y mostrado el firme propósito de llegar al objetivo sin retrasos injustificables a la vez que garantizado la seguridad física de los arsenales pendientes. Por el lado negativo, la distribución de recursos económicos y financieros dentro de las inspecciones por rutina se ha centrado fundamentalmente en el desarme, y menos en la no-proliferación ${ }^{34}$. En este sentido, a medida que el proceso de destrucción va avanzando, el principal riesgo tradicional causado por la existencia de ingentes arsenales de armas químicas en las dos superpotencias ha pasado más bien a un segundo plano por ser un riesgo bajo relativo control, con lo que debería requerir menos concentración de recursos.

No obstante, estructuralmente hay un énfasis en el concepto tradicional de aproximación a las inspecciones, por el riesgo clásico de los agentes conocidos, en detrimento de las inspecciones a instalaciones y compuestos más avanzados tecnológicamente que, en un campo en continúo avance como la química, pudieran presentar un mayor riesgo. En este punto, se ha mostrando un desequilibrio claro entre el numero de inspecciones destinadas a las

de las declaraciones industriales fue identificada en la Primera Revisión como una de las áreas prioritarias de mejora del régimen de no proliferación química.

${ }^{33}$ Mientras en el año 2000, la Secretaría Técnica solicitó aclaración de 88 Estados Partes, y en abril del siguiente año solamente el $46 \%$ respondió a tal, en el año 2008, el $86 \%$ de los países había enviado sus declaraciones a tiempo, incluidas las declaraciones anticipadas de actividades para el año 2009. Conference of States Parties: "Draft Report of the OPCW on the Implementation of the Convention in 2008", C-14/CRP.1 (30 Nov. -4 Dec. 2009), p. 8.

34 "Note by the Director-General Summary of Verification Activities in 2009", OPCW Document, S/869/2010. 
listas tradicionales (listas 1, 2 y 3) respecto las OCPF (Other Chemical Production Facilites $)^{35}$. Para permitir una mayor adaptación a los futuros agentes y procedimientos de producción, así como garantizar una efectiva y completa implantación de la norma de inspección en un contexto cambiante, tanto técnicamente como bajo el punto de vista de la amenaza, es necesario que se exploren nuevas aproximaciones de inspección a las instalaciones industriales, particularmente a las OCPF de lo contrario, puede quedar mermada la eficacia del régimen de las armas químicas. Las nuevas aproximaciones deben de tener en cuenta no solamente las características de las nuevas tecnologías y avances de la ciencia ${ }^{36}$, sino también la migración geográfica de los focos de riesgo ${ }^{37}$. En suma, la materialización del principio de Criterio General del Propósito en la norma de inspección y sus respectivas reglas queda desequilibrado en cuanto a los agentes clásicos respecto otras amenazas en el ámbito industrial $^{38}$. En este sentido, la UE y sus Estados miembros consideran las OCPF como un desafío al objeto y propósito de la Convención y han ejercido considerable influencia en cambiar esta tendencia ${ }^{39}$, no obstante, el cambio cualitativo no ha venido acompañado de un cambio cuantitativo, ya que el numero de inspecciones OCPF es bajo comparado con el número de instalaciones, siendo solamente un $3 \%$ del total de las instalaciones inspeccionables ${ }^{40}$.

A diferencia de la norma de destrucción o la de no proliferación, la Convención no contiene reglas específicas que desarrollen la norma que prohíbe la preparación, planeamiento o la inducción al delito. Todas estas actividades se consideran como prohibidas, y se verifican subsidiariamente mediante el sistema de inspecciones por rutina, el control de las declaraciones y las inspecciones por denuncia. Así, el régimen de verificación de las armas químicas también contempla la posibilidad de lanzar inspecciones por denuncia que sirvan para restaurar la confianza y demostrar el cumplimiento. A este respecto, la consideración de las Inspecciones por Denuncia como un elemento del más alto nivel político y susceptible de ocasionar desprestigio internacional, o perjuicio a la credibilidad del régimen, ha provocado un importante efecto de disuasión a favor de la estabilidad del mismo. En contrapartida, la estabilidad creada por la disuasión puede llevar a la atrofia del instrumento, aunque la realización de inspecciones de entrenamiento sirva para adquirir o mantener cierta capacidad

\footnotetext{
${ }^{35}$ Otras Instalaciones de Producción de Sustancias Químicas, que producen que una sustancia química orgánica definida no incluida en las Listas que contenga los elementos fósforo, azufre o flúor.

${ }^{36}$ Green, Nick: "Advances in Science and Technology Relevant to BCW and CWC", presentado en la conferencia: New Approaches to WMD proliferation, The Royal Society, 2 de mayo 2006; Lowe, Holger; Hessel, Volker y Mueller, Andreas: "Microreactors. Prospects already achieved and possible misuse", Pure Appl. Chem., vol.74, $\mathrm{n}^{\mathrm{o}} 12$ (2002), pp. 2271-2276. También en: Kelle, Alexander (ed.): "The Changing Scientific and Technological Basis of the CBW Proliferation Problem", A Workshop Report Preventing the Misuse of 21st Century Chemistry: State of the Art of Drug, Development and Delivery, and Selected Enabling Technologies, Belfast (Enero 2006); Trapp, Ralf: “Advances in Science and Technology and the Chemical Weapons Convention”, Arms Control Today, Marzo 2008. Balali-Mood, Mahdi; Steyn, Pieter, S.; Leiv K. Sydnes y Trapp, Ralf: "Impact of Scientific Developments on the Chemical Weapons Convention", Pure Appl. Chem., vol. 80, $\mathrm{n}^{\mathrm{o}} 1$ (2008), pp. 175-200. Además, la OPAQ, con el apoyo de la UE, organizó un Foro Académico y un Foro para la Industria y Protección para investigar a los cambios estratégicos para la Convención: "Note by the Technical Secretariat, The 2007 OPCW Academic Forum and the Industry and Protection Forum: In Support of Comprehensive Implementation of the Chemical Weapons Convention", Office of Special Projects S/674/2008, 1 Febrero de 2008.

37 Tucker, Jonathan B.: "Verifying the Chemical Weapons Ban: Missing Elements", Arms Control Today, (enero-febrero de 2007), p. 4. También en: "Preparing for the Second CWC Review Conference: HSP Views", The CBW Conventions Bulletin, $\mathrm{n}^{\circ} .76+77$ (Octubre 2007), p. 1.

${ }^{38}$ Tucker: "Verifying the Chemical Weapons Ban...", op. cit., pp. 1-3.

${ }^{39}$ La comparación de inspecciones realizadas entre las lista tradicionales/OCPF fue de 32/100 en el año 2001 pasando a 83/125 en el año 2010.

${ }^{40}$ OPCW Conference of State Parties, C-16/4, 11 de Noviembre 2010.
} 
operativa, éstas deberían realizarse con mayor frecuencia y más implicación de los Estados Parte, particularmente los no occidentales. En este sentido, a pesar de la clara postura de la $\mathrm{UE}^{41}$ a favor de la utilización funcional del instrumento como en otros tratados de armamento convencional (FACE, DV 99), el asunto permanece sin avance y hasta el momento las Inspecciones por Denuncia solo han sido lanzadas como ejercicios.

La universalidad ha sido y es una prioridad clave de la CAQ. Muchos de los reductos clave se encuentran en el Oriente Medio y, habida cuenta de las tensiones de la región y la historia, se encuentran entre los más propensos a usar las armas químicas. La ausencia de incluso pequeños estados de la CAQ podría socavar el tratado, proporcionando refugios o puntos de transbordo para los actores no estatales y las redes de contrabando. Sin embargo, en el actual contexto de seguridad internacional, la amenaza principal ha surgido en la cuenca mediterránea por aquellos Estados fallidos como consecuencia de las revoluciones de "la primavera árabe" que, además, son poseedores de armas químicas bien sean firmantes de la CAQ (Libia) o no (Siria). Los riesgos principales son de tres tipos, en primer lugar, que las tropas lealistas utilicen las armas químicas contra los revolucionarios como arma de último recurso, segundo, que se pierda el control de las armas químicas y caigan en manos de terroristas o delincuencia organizada, tercero y último, que durante el combate se produzca una explosión sobre los almacenes que libere armas químicas y pueda afectar a la población civil.

Aunque el riesgo es inevitable durante el periodo de combates, el problema de las armas químicas en estados fallidos que son parte de la CAQ es menor, principalmente porque ya se han establecido inventarios previos, estándares de control $^{42}$, incluso, en caso de estar afectada población civil, se pueden ejecutar mecanismos de asistencia previstos en el Art. X. además, en el caso de Libia, la revolución ha servido para descubrir nuevas existencias no declaradas $^{43}$. En el caso de estados fallidos no firmantes, el problema se aumenta por la falta de información previa de las existencias, de forma que cualquier tipo de perdida o comercio de armas químicas pudiera no quedar registrado al no existir inventarios efectuados por un organismo independiente. Finalmente, el uso como recurso desesperado, aunque menos probable debido a la fuerte presión internacional no es descartable ${ }^{44}$.

La adhesión universal no solamente fortalecería la norma contra las armas químicas mediante la demostración de que este principio es aceptado en muchos entornos diferentes políticos, culturales, religiosos, económicos y legales. Por otra parte, el número de estados que se adhieren a un tratado es un criterio utilizado para determinar si se forma una regla de derecho internacional consuetudinario y por lo tanto es de obligado cumplimiento para todos los estados sean parte o no se hayan adherido. La CAQ entró en vigor en 1997 y, desde entonces, la membresía de la CAQ se ha incrementado de manera constante y se está acercando a la universalidad. En particular, el número de los estados partes ha aumentado más rápidamente después de la Primera Conferencia de Revisión en 2003, la aprobación un Plan

\footnotetext{
${ }^{41}$ The Hellenic Republic on Behalf of the European Union: "Challenge Inspections: Views of the European Union”, The EU working paper, OPCW document RC-1/NAT.21 (1 de Mayo 2003).

${ }^{42}$ Webb, Sarah: "Libyan chemical weapons stockpile intact: inspectors", Reuters, 4 de Noviembre 2011, en www.reuters.com.

43 BBC: "Libya's Muammar Gaddafi had chemical weapon cache", BBC News, 20 de Enero 2012, en www.bbc.co.uk.

44 BBC: "Syria chemical weapons: Obama says world is watching", BBC News, 23 de Julio 012, en www.bbc.co.uk.
} 
de Acción ${ }^{45}$ y las Acciones Conjuntas con las UE el número de estados fuera de la CAQ se ha reducido de 40 en 2003 a 12 en 2007 y a 7 en el 2012.

\section{La política europea de las armas: efectividad y coherencia}

Como hemos señalado, desde la creación de la Estrategia ADM hasta la actualidad, la política de la UE respecto a las armas químicas ha estado dividida en dos periodos, cuyo punto de inflexión es la creación de las Nuevas Líneas de Acción de la UE en el 2008. Es importante señalar que la Estrategia $\mathrm{ADM}$ es un documento que todavía sigue vigente aunque su implantación ha sido objeto de dos diferentes dinámicas.

Durante el primer periodo, la acción de la UE en el ámbito de las armas químicas se ha materializado únicamente en Acciones Conjuntas con la OPAQ (a excepción de ayudas puntuales al programa de destrucción Federación Rusa). La característica principal de este periodo ha sido casi exclusiva dedicación de las acciones a fomentar la universalidad (en primer plano) y la implantación nacional. Así, la distribución de fondos entre los proyectos mostraba plenamente la política de poder blando de la UE, dedicando la mayor parte de los fondos a los proyectos de cooperación internacional e intercambio científico. Sirva de ejemplo los fondos destinados a la adquisición de equipos básicos (PC's) con los que poner en marcha las Autoridades Nacionales y, principalmente, impulso de las capacidades de análisis químico, lo que, además de implicar asistencia tecnológica sirve para mejorar la capacidad de vigilancia $^{46}$.

En este sentido, su principal objetivo, la promoción de la universalidad, resultó ser bastante efectiva, aunque con ciertas consideraciones. Por un lado, aunque no se pueda justificar completamente una relación causa- efecto (attribution gap $^{47}$ ), por la cual el apoyo de la UE tuviese como consecuencia directa el aumento de la membresía, es cierto que, en los años $2005^{48}$ y $2006^{49}$ se produjo un aumento sustantivo de las membresías que formaron parte de los programas de universalización conjunta UE-OPAQ. Con el apoyo de la UE el aumento del membresía de la CAQ sumó 14 Estados alcanzado los 181 Estados Parte. Por el otro lado, aunque los nuevos Estados Parte no supusieran en si mismo un sustantivo riesgo para la CAQ, al no tener ni capacidad indígena de producción, ni poseer armas químicas, no cabe duda que su adhesión contribuye a disminuir el número de santuarios fuera del régimen de las armas químicas que pueden servir de amparo a los proliferadores.

En el año 2007, como consecuencia del "documento de reflexión” y la creación se produjeron los primeros cambios en la asignación de fondos a programas de asistencia contra ataques con armas químicas. Resulta complicado evaluar la efectividad real de los cambios, por dos motivos principales. Por un lado, la dificultad de valorar el impacto de la financiación sobre proyectos con resultados parcialmente mesurables hasta su excepcional puesta en práctica como en el caso tanto del mecanismo de asistencia y protección, como adquisición de capacidades nacionales al ser víctima de un ataque con armas químicas. Por el otro lado, la imposibilidad de aplicar una metodología a otros de carácter más relativo, como el proyecto de amentar la concienciación parlamentaria respecto al establecimiento legal de la CAQ. Todo

\footnotetext{
${ }^{45}$ OPCW Document, EC-M-23/Dec.3, 24 de Octubre 2003.

${ }^{46}$ Council of the European Union, Council Joint Action 2004/797/CFSP, op. cit., p. 67.

${ }^{47}$ Grip, “Assessing Selected European Union...”, op. cit., p.18.

${ }^{48}$ Antigua y Barbados, Granada, República Democrática del Congo, Honduras y Niue.

${ }^{49}$ República Centro Africana, Islas Comores, Djibouti, Montenegro y Haiti.
} 
ello, sin caer en el tópico de asociar directamente dinero gastado con propósitos conseguidos ${ }^{50}$.

Sin embargo, las primeras iniciativas en el ámbito de la Estrategia de ADM, incluidas las de las armas químicas, todavía mostraban carencias importantes, principalmente de falta de coherencia ${ }^{51}$, la necesidad de recursos adicionales para proyectos de destrucción de arsenal química en la Federación Rusa y, finalmente, la implantación de la CAQ en algunos Estados Parte $^{52}$. En este sentido, es significativo señalar la doble vertiente del concepto coherencia, que implica tanto la complementariedad de las actividades de la UE, actuales y en proyecto, respecto de las prioridades políticas establecidas por la Estrategia ADM y el Consejo (coherencia de los programas de ayuda), como la cohesión entre los diversos instrumentos de política exterior de la Unión y la cooperación entre las instituciones de la UE, y entre éstas y los Estados miembros ${ }^{53}$.

No obstante, varios puntos son destacables respecto a la efectividad, por lado negativo, la ampliación de proyectos no llevó consigo incremento de la financiación respecto Acciones Conjuntas previas, disminuyendo así las asignaciones a partidas como el fomento de la universalidad que vieron disminuido su presupuesto a casi la mitad, aunque siguieron teniendo relativo éxito ${ }^{54}$. Por el lado positivo, por primera vez, los resultados serian evaluados por las instituciones competentes de la UE, para este propósito, la OPAQ debía proporcionar a la Presidencia de la UE, través del Alto Representante/Secretario General y a la Comisión informes detallados de la implantación.

Revisando los propósitos, iniciativas y actividades de la UE, con los objetivos alcanzados durante este primer periodo, se puede concluir que el multilateralismo, más concretamente la potenciación de la universalidad, ha centrado todos los esfuerzos de la UE en este sentido, dejando a los otros pilares de la Estrategia, la prevención y la cooperación internacional, reducidas al mínimo. De forma general, los resultados más visibles de la cooperación con la OPAQ se han obtenido en el ámbito de la universalidad. Hasta la actualidad, la UE ha financiado a la OPAQ con más de 7 Mill. de euros en diferentes proyectos, mediante el apoyo a seminarios regionales para promover la universalización de la CAQ y, como hemos tenido ocasión de señalar, aumentando el número de adeptos en África, el Mediterráneo, Oriente Medio y el Caribe ${ }^{55}$. Más tarde, el apoyo a la OPAQ para mejorar la universalidad ha priorizado las visitas bilaterales (en vez de seminarios regionales) con un enfoque particular en los países africanos. Además, la acción específica para la universalización de CAQ se ha completado con acción diplomática de la Presidencia de la UE y los Estados miembros mediante gestiones bilaterales ante los distintos países para

\footnotetext{
${ }^{50}$ Van Ham, op. cit., p. 9.

${ }^{51}$ House of Lords, European Union Committee: "Preventing Proliferation of Weapons of Mass Destruction: The EU Contribution Report with Evidence", 13th Report of Session 2004-05, Stationary Office, Londres, (Abril 2005), p. 28.

52 SIPRI: "PILOT PROJECT, Strengthening European Action on WMD Non-Proliferation and Disarmament: How Can European Community Instruments Contribute?", Interim report, Estocolmo, (Noviembre 2005), pp. 28-32.

${ }^{53}$ Council of European Union, "EU Strategy against the proliferation of WMD: Monitoring and enhancing consistent implementation" (12 de Diciembre 2006), pp. 3, 6.

${ }^{54}$ Entre los años 2007 y 2008, firmaron cuatro Estados que, a su vez, formaron parte los proyectos conjuntos de universalidad de la UE: el Congo, Guinea Bissau, Líbano y Barbados.

${ }^{55}$ Entre 2008 y 2012 accedieron Bahamas, Congo, la Republica Dominicana, Guinea Bissau, Irak y Líbano. Dejando el número de países no signatarios en seis: Angola, Egipto, República Popular Democrática de Corea, Somalia, Siria y Sudan. Signatarios pero no ratificados: Israel y Myanmar. www.opcw.org (consultado 29 Julio 2012).
} 
convencer a ratificar la CAQ y poner en práctica una completa implantación de sus disposiciones ${ }^{56}$.

Por el lado negativo, durante este periodo la UE no ha llevado a cabo acciones específicas resolutorias para compensar las vulnerabilidades del régimen de la $\mathrm{CAQ}$, tanto en el seno de la OPAQ, como actor independiente. En este sentido la UE parece que ha trabajado más la idea de la universalidad como representación y paradigma del multilateralismo, enfocando su esfuerzo únicamente en el apoyo al régimen existente de la CAQ, que a incrementar la efectividad de la propia Estrategia de la UE mediante el esfuerzo sus tres principios y la consecución de sus propios objetivos como actor internacional.

No obstante, los primeros avances significativos hacia la consecución de los objetivos de la Estrategia se han producido con el establecimiento de las Nuevas Líneas de Acción en el 2008. Así, aunque estas fueran creadas por la necesidad de revisar la Estrategia de la UE, o desde un punto de vista mas pragmático, como llamada de atención a los Estados Parte a cumplir los compromisos en el $2003^{57}$, han supuesto un considerable avance por tres motivos principalmente. En primer lugar, han implicado un cambio sustancial en la aproximación al problema de la proliferación de las armas químicas, pasando de una acción caracterizada principalmente por el "multilateralismo efectivo", a diversas acciones de carácter más operativo, definidas en términos de objetivos a ser logrados y capacidades a ser adquiridas. Además, establece acciones que obligan a ir más allá del apoyo al régimen de la CAQ, como el establecimiento de sus propios Centros de Excelencia QBRN. En segundo término, supone un incremento presupuestario del $25 \%$ respecto las contribuciones anteriores a la OPAQ. Finalmente, la creación de un Consorcio de No Proliferación, que sirve de apoyo académico a los órganos de decisión de la UE.

El carácter positivo de esta evolución queda empañado por varios hechos. En primer lugar, la lentitud del proceso, habiéndose establecido finales del 2010 para implantar las medidas, se tuvo que extender el plazo hasta diciembre del 2012. Segundo, la necesidad de avanzar en las prioridades transversales (cross cutting priorities) como el control de visados y programas de tanto de concienciación como de códigos de conducta de la comunidad científica e industrial sensible de ser manejar sustancias de doble uso. Resulta significativo que las áreas menos desarrolladas por las Nuevas Líneas de Acción coincidan con aquellas donde el régimen de la CAQ se muestra mas débil o no tenga mandato para poder ejercer su efectividad. En este sentido se explica la encrucijada donde se encuentra la UE, donde las vulnerabilidades de la OPAQ no le dejan otra elección que implantar de manera efectiva su propia Estrategia ADM.

\section{Conclusiones}

En 2003, la decisión de la UE de adoptar un enfoque basado en el "multilateralismo efectivo" se basaba en tres elementos: fomento el multilateralismo del sistema de tratados internacionales; promoción de un entorno regional e internacional estable y estrechamiento de los lazos con los países aliados. No obstante, durante el periodo de aplicación inicial hasta el año 2008, la materialización en Líneas de Acción consistió, casi exclusivamente (con

\footnotetext{
56 Giannella, Annalisa: "EU Action in support of OPCW activities 2005-2008: effective multilateralim in practice", Second Special Session of the Conference of the State Parties to Review the Operation of the CWC, EU side event, La Haya, (14 de Abril 2008), pp.4-7.

${ }^{57}$ Van Ham, op. cit., p. 13.
} 
excepción de las ayudas a la Federación Rusa), en el impulso al primer elemento mediante sucesivas contribuciones económicas a la OPAQ. Aunque la contribución fuese coherente con la política de poder blando y cooperación económica característica de la UE, y con resultados positivos en cuanto de la universalidad (incluida la cláusula de no proliferación), no tuvo peso específico en resolver las cuestiones pendientes que erosionan la efectividad de la CAQ, como la implantación nacional y refuerzo de las Autoridades Nacionales, la verificación industrial y los avances de la ciencia, el desequilibrio entre desarme y no proliferación, el uso funcional de las inspecciones por denuncia o el control del comercio y exportaciones de sustancias sujetas a control del régimen.

Las Nuevas Líneas de Acción del año 2008 han provocado que la UE tomase medidas propias como actor internacional (sobre todo respecto al control del comercio y las exportaciones, así como la protección contra armas químicas) compensando, en cierta medida, algunas de las carencias de las líneas de acción iniciales, y del régimen de la CAQ. Sin embargo, la casi exclusiva referencia de la Unión Europea a la OPAQ hace que las vulnerabilidades de ésta, sobre todo frente al cambio del contexto internacional de seguridad y los avances de la ciencia, puedan colocar a la UE en detrimento del su propia estrategia inicial. Por el propio interés de la UE, resulta necesario observar cuidadosamente la evolución del régimen de la CAQ, particularmente después de haber casi completado la destrucción de ingentes arsenales iniciales de las superpotencias, y el desvío de la amenaza hacia geografías, tecnologías y escenarios diferentes, para decidir si la UE debe reforzar sus acciones autónomas como actor internacional. 